UCRL-JC-118659

PREPRINT

\title{
Open-Ended Magnetic Confinement Systems for Fusion
}

\author{
Richard F. Post \\ Dmitri D. Ryutov \\ Lawrence Livermore National Laboratory \\ Livermore, California
}

This paper was prepared for submittal to

The Conference Proceedings of the International Symposium on

Evaluation of Current Trends in Fusion Research

Washington, D.C.

November 14-18, 1994

May, 1995

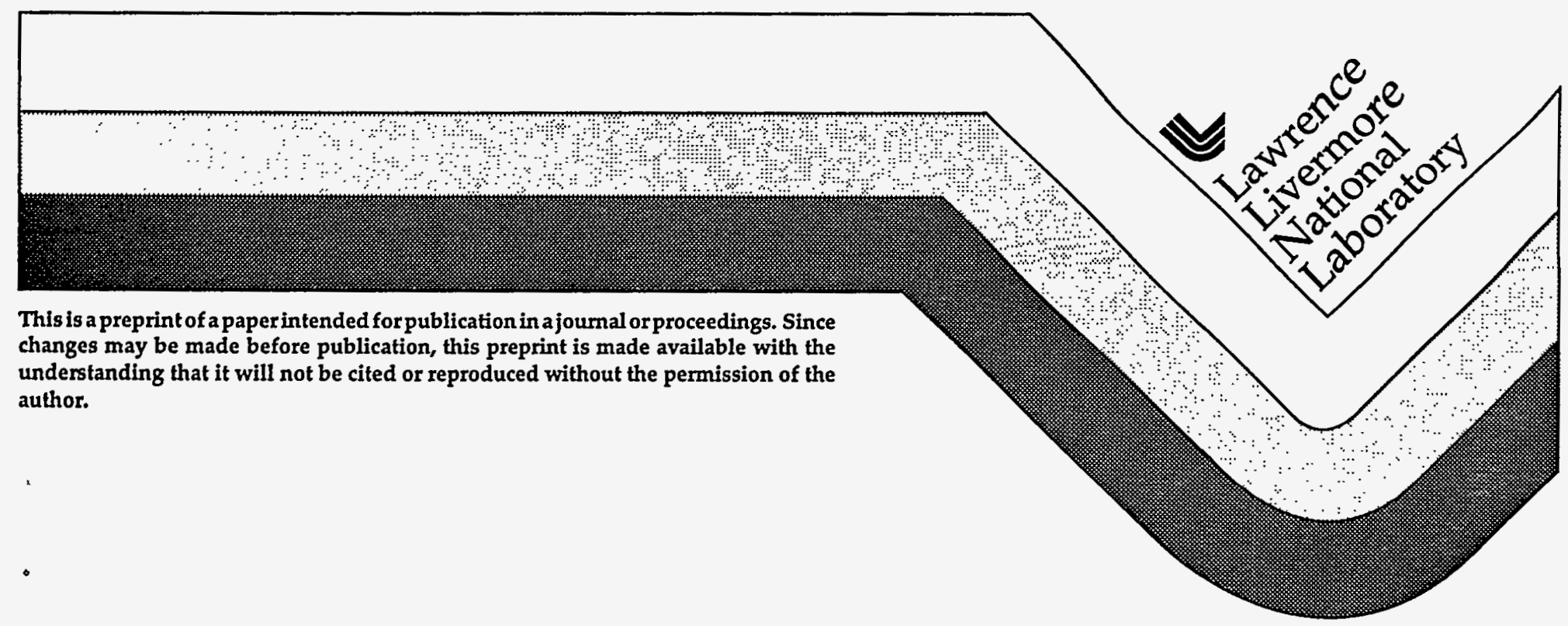

RECEIVED

AUG 041995

OSTI 


\section{DISCLAIMER}

This document was prepared as an account of work sponsored by an agency of the United States Government. Neither the United States Government nor the Uni versity of California nor any of their employees, makes any warranty, express or implied, or assumes any legal liability or responsibility for the accuracy, completeness, or usefulness of any information, apparatus, product, or process disclosed, or represents that its use would not infringeprivately owned rights. Reference herein to any specific commercial products, process, or service by trade name, trademark, manufacturer, or otherwise, does not necessarily constitute or imply its endorsement, recommendation, or favoring by the United States Government or the University of California. The views and opinions of authors expressed herein do not necessarily state or reflect those of the United States Government or the University of California, and shall not be used for advertising or product endorsement purposes.

\section{3)}

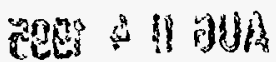

त 


\section{DISCLAIMER}

Portions of this document may be illegible in electronic image products. Images are produced from the best available original document. 


\title{
OPEN-ENDED MAGNETIC CONFINEMENT SYSTEMS FOR FUSION*
}

\author{
Richard F. Post and Dmitri D. Ryutov \\ Lawrence Livermore National Laboratory \\ Livermore, CA 94550
}

USA

\begin{abstract}
Magnetic confinement systems that use externally generated magnetic fields can be divided topologically into two classes: "closed" and "open". The tokamak, the stellarator, and the reversed-field-pinch approaches are representatives of the first category, while mirror-based systems and their variants are of the second category. While the recent thrust of magnetic fusion research, with its emphasis on the tokamak, has been concentrated on closed geometry, there are significant reasons for the continued pursuit of research into open-ended systems. The paper discusses these reasons, reviews the history and the present status of open-ended systems, and suggests some future directions for the research.

\section{(I) Introduction}

One obvious way to differentiate between classes of magnetic confinement systems that use externally generated magnetic fields is via the topology of their fields. Here by "the topology of the field" we mean whether the field lines of the externally applied field are "closed", i.e., are "endless" in that they are contained within a toroidal confinement region (as in a tokamak), or "open", that is, leave the plasma confinement zone at either end of an essentially linear system (for example, as in a magnetic mirror system).

It should be noted that the distinction that we have adopted here between "open" and "closed" systems, since it relates to the topology of magnetic fields generated by external coils, includes in the class of open systems not only mirror machines but FRC's (Field-Reversed Configurations), and RFM's (Reversed-Field Mirrors), both of which share the same topology of the externally generated field. Thus, in the discussion to follow, many of the points made apply to these other types of open systems.
\end{abstract}


The seemingly innocuous topological distinction between closed and open systems actually has profound consequences, both in the plasma physics issues involved and in the paths available toward economic fusion power systems. As an example, in closed systems particle losses, occurring only across the magnetic field lines, result necessarily in major radial variations in the plasma parameters. These include, for example, negative temperature gradients as the plasma approaches the chamber wall. Such temperature gradients, communicated back into the plasma, have been shown to be a source of plasma instabilities [1]. Such instabilities can lead to cross-field diffusion rates far in excess of those expected for a quiescent plasma. By contrast, in open-ended systems where the particle losses are out the ends, there is no requirement for contact of the plasma with the walls of its confining chamber, thus no enforced radial temperature gradient need be present. As a result the radial confinement properties of open-ended systems can in principle be superior to those of closed systems.

The comments in this paper are aimed at a discussion of some of these topologically related differences between the two systems. It is hoped that one of the byproducts of such a discussion will be to encourage fusion researchers to take a broader view of the magnetic fusion problem than the more usual one, the one that contends that the main criterion by which to compare approaches is "energy confinement time". By extension, energy confinement time is related to the attainable fusion energy-multiplication factor, Q, (ratio of fusion power to power required to heat and sustain the plasma) i.e., it determines the $Q$ value that can be achieved. In the final analysis, capability for attaining a high $Q$ is only one of several criteria for judging the economic potential of a magnetic confinement approach. High attainable $\mathrm{Q}$ may, in fact, turn out to be secondary to other criteria that are more significant in the context of comparative economic viability.

The thesis will be advanced that there are many probable economic and other advantages of open systems over closed ones that at the very least justify a continued search for ways to exploit these advantages. This line of reasoning is intended to apply especially to those ways that put minimal demands on the weakest feature of some open-ended systems, namely their in-some-cases low $Q$ values, as exemplified by the simple mirror machine with its well-known end losses. We will, therefore, pay special attention to low-Q systems, their properties and their virtues. By relaxing the (sometimes myopic) concentration on achieving high $Q$ there is opened up a much wider 
variety of possibilities for the confinement system itself. It is entirely possible, we would say even likely, that within this wider range of possibilities there exist systems that are better suited to developing into economically viable fusion power systems than is the tokamak.

After all, the ultimate goal of fusion is not the simplistic one, $Q=$ $\infty$, but rather it is the pragmatic one, "to generate power at a competitive cost, and with superior environmental and safety characteristics as compared to other energy sources". As the search for practical fusion power proceeds towards its goal, we must pay increasing attention to the pragmatic goal, which may or may not involve a quest for ever-higher $Q$ values.

Specifically, our intent here is to state the case for broadening the attention of the fusion community to issues beyond a search for everhigher $Q$ values, and to encourage a look at low- $Q$ systems for their potential advantages. In a later section we will sketch out a hypothetical "fusion power system" where the goals are, first, to use the D-He3 fusion cycle with direct conversion of the fusion energy to electricity, and second, to operate in a plasma regime that permits the near-suppression of satellite neutron-producing D-D reactions. To accomplish both of these goals it will be seen that it is essential to operate at as low a $Q$ value as is practical. High-Q operation is counterproductive of the stated goals.

\section{II) Brief review of the history of open-ended systems}

In surveying the long history of mirror research up to the present time one is struck by the evident fact that, as contrasted to closed systems, there has always existed a close relationship between theory and experiment. Mirror systems have not only been found to be tractable to analysis, but this analysis has also pointed the way to the achievement of situations that have rarely if ever been seen in closed systems: We refer to plasmas that are so near to the quiescent state that their confinement can be predicted on the basis of classical processes, i.e., collision-related phenomena, as opposed to the turbulence-related effects that dominate the confinement in closed systems.

As we have noted earlier, open-ended confinement systems must face a problem not encountered in closed-field systems, namely, plasma losses along the field lines, out the ends of the system. The mirror machine represents one way of attacking this problem in that it utilizes 
the magnetic mirror effect, arising when a particle spiraling along field lines encounters, and is reflected back, by an increasing magnetic field. We can therefore trace the historical origin of mirror systems to the study of particle mirroring in connection with cosmic ray physics, analyzed early on by Störmer [2]. His studies concerned the reflection of incoming cosmic rays (protons, etc.) by their encounter with the earth's magnetic field. Many years later, in the discovery of the "Van Allen belts" [3] of charged particles magnetically trapped between the earth's north and south magnetic poles mirror confinement of charged particles was seen on a grand scale, some years after it was first studied in the laboratory (in the early 1950's).

The theory of particle confinement in mirrors, as controlled by socalled "adiabatic invariants: was elegantly derived by Teller and Northrop [4]. It was proved out in the laboratory and in a spectacular space-related experiment. In the laboratory experiments, for example one in 1962 by Gibson, Jordan, and Lauer [5], positrons from radioactive neon were trapped between mirrors and contained for times of order of seconds, corresponding to millions of reflections. Their loss occurred only as the result of collisions with background gas atoms, not as a result of some failure of the mirror process. In the "Argus" experiment in space, proposed by Christofilos and carried out in 1958, a small atomic bomb, sent into the earth's magnetosphere by rocket, was detonated, producing a cloud of energetic electrons from beta decay. These electrons, trapped between the earth's magnetic poles, spread out into a thin shell surrounding the earth. A detectable fraction of these electrons was still present decades after the detonation!

The basic principle behind both of these experiments is that particle confinement in a mirror machine is governed by "adiabatic invariants", the constancy of which assures that charged particles, once trapped between mirrors, will remain trapped essentially "forever" unless some random process, for example inter-particle collisions, perturbs their motion. There are only two conditions that the particles must satisfy in order to remain trapped. The first requirement is that the size of their gyro orbit around the field lines must be "small" (i.e. less than of order 10 percent) compared to the characteristic gradient lengths of the confining field. This requirement is not difficult to satisfy practically as it simply puts lower bounds on the size of the apparatus and/or the intensity of the confining field.

The second requirement for particles to be mirror confined is that the pitch angle the particles' spiralling orbits make with the field line 
direction at the midplane between the end mirrors must be larger than the "loss cone" angle, $\theta_{\mathrm{LC}}$, defined through the relationship

$$
\operatorname{Sin}\left(\theta_{L C}\right)=R^{-1 / 2}
$$

where $\mathrm{R}=\mathrm{B}_{\max } / \mathrm{B}_{\min }$ is the so-called "mirror ratio", i.e. the ratio of the magnetic field intensity at the mirrors to that at the minimum point (the midplane).

The two adiabatic invariants which, together with the law of energy conservation $\mathrm{E}=$ constant, govern mirror confinement are: (1) the magnetic moment, $\mu=\mathrm{w}_{\perp} / \mathrm{B}$ (given by the energy associated with the cyclotronic motion of the particle around the field line on which they are moving divided by the local value of the magnetic field), and, (2) the action integral, $\mathrm{J}$, carried out over the longitudinal motion along the field lines between the mirroring points. The first of these determines whether or not the particle is trapped between the mirrors; too small a value of $w_{\perp}$ relative to the energy of motion parallel to the field line will put the particle into the loss cone so that it is immediately lost upon its next encounter with the mirror. As will be described, the second invariant, $\mathrm{J}$, determines the transverse dimension of the drift surface on which the particle moves, thus whether or not it will hit the chamber wall in the course of bouncing between the mirrors.

As a consequence of these two invariants trapped particles move back and forth between the mirroring points, while at the same time drifting transversely, but in such a way that, for a properly chosen class of magnetic geometries, the "guiding center" of their spiraling motion generates a closed drift surface surrounding the centermost field line. Then, once trapped between the mirrors, particles remain trapped until some time-varying process, such as collisional interactions, sufficiently perturb their energy and one or the other of the two invariants to destroy trapping. Perturbations of $\mathrm{E}, \mu$, and $\mathrm{J}$ lead to leakage out the mirrors and to diffusion between drift surfaces.

It is to be noted that these invariants operate just as effectively in non-vacuum magnetic fields, that is, magnetic fields that are the resultant of external coils and of diamagnetic currents flowing in the plasma, provided only that the non-vacuum field also satisfies the stated requirements for adiabaticity. 
Thus, in seeking to understand the differences between open and closed systems it is important to recognize that mirror systems start with an external field that guarantees almost indefinitely long particle confinement in the absence of perturbing effects. Furthermore, confinement will still be effective provided the "perturbing effects", such as steady diamagnetic currents in the plasma, do not vitiate the conditions for adiabaticity. By contrast, a closed system such as the tokamak starts with an external field that cannot contain particles in long term (because of transverse drifts associated with the toroidal field). In order to achieve long-time confinement it must rely on currents flowing in the plasma to overcome these drifts.

Returning to the early history of mirror confinement, it began with close contact with theory, in the form of the theory of adiabatic invariants, and then proceeded toward its encounter with plasmainduced effects. The first of these, a sword of Damocles that would hang over it throughout its progress, is the effect of particle collisions on the confinement, and the particle leakage out the ends that these collisions can cause.

Since collisions can affect the magnetic moment and particle energy, they can have an immediate effect on mirror trapping, i.e., on containment. That is to say, it will take only about one "effective collision", i. e., a deflection of the pitch angle through 90 degrees, to cause a trapped particle to enter the loss cone, and thus be lost upon its next encounter with an end mirror. This situation is to be contrasted with the one associated with closed systems, such as the tokamak. In closed systems, where the only escape route for the particles is across the field lines to the chamber wall, collisions lead to a random walk process in which many collisions may be required before the particle reaches the wall. Furthermore, as long as this process is a diffusion-like one, as opposed to a convective loss, the confinement time will be found to scale roughly as the square of the radius of the chamber, so that even in the presence of turbulent processes long confinement times can be achieved, though at the price of making the chamber dimensions very large.

Early on calculations were made, utilizing the Fokker-Planck (F-P) equation, of the confinement time against mirror losses of conventional (two-mirror) mirror machines. The first such calculations did not include an important effect: the electric potential (ambipolar potential) that arises naturally because of the disparity in scattering rates of electrons and ions (electrons scatter more rapidly). Since on the average 
the electron and ion loss rates must balance in steady state, a positive potential, of the plasma relative to the outside world, appears as soon as the loss rate reaches steady-state. This positive potential, trapping for the electrons and detrapping for the ions, somewhat accelerates the rate of loss of the ions over the value calculated in the absence of the potential. Thus it was, in the earliest calculations, the confinement time, though not robustly long, seemed adequate to achieve net fusion power, if the ion temperature was high enough. The first F-P code calculations were made in the 1950's, and by 1959 the codes had been refined to the point that all important effects were being included. As an example, the work of Kuo-Petravic, Petravic, and Watson [6] gave for the $n \tau$ containment parameter (for a D-T-electron plasma) the scaling relationship:

$$
\mathrm{n} \tau=2.33 \times 10^{10} \mathrm{E}_{0}^{3 / 2} \log _{10}(\mathrm{R}) \mathrm{cm}^{-3} \mathrm{sec} .
$$

where $\mathrm{E}_{0}$ is the mean ion energy in $\mathrm{keV}$. As can be seen immediately by putting in the numbers, to achieve $n \tau$ values leading to $Q$ values in excess of 1.0 would require mean ion energies of order $100 \mathrm{keV}$ and mirror ratios of 3 or more. This result put the simple mirror machine in the category of low $\mathrm{Q}$ approaches ab initio. It thereby sharpened the need for innovative improvements in order to make it a viable candidate for a fusion power system. We shall later discuss some of these improvements, following the mention of two other problems for mirrors, ones that were addressed and solved with great success.

The first of these problems, pointed out early on, during the classified days of fusion research (circa 1954) by Edward Teller, was that of MHD stability of the equilibrium. In a simple mirror machine, as shown in Fig (1), the field lines between the mirrors are concave inward, implying a negative field gradient in the radial direction. This "bad curvature" region is one that is therefore potentially subject to MHD instabilities, i.e., to unstable transverse drifts of the plasma. In the earliest mirror experiments no such drifts were seen, for reasons that were not then understood, and the problem was put out of mind. In fact, in some experiments at Livermore where a "hot electron" plasma was created by magnetic compression in a pulsed mirror field, [7] not only was a stable plasma column observed, but radial diffusion rates were measured that were five orders of magnitude slower than the rate predicted for "Bohm" diffusion as observed in some closed-geometry systems. However, with progress in generating hot ion plasmas, ones better isolated from plasmas outside the mirrors, unstable transverse 
drifts of the plasma were seen, in agreement with the MHD predictions. There was indeed a problem, one that demanded a solution.

In 1961, in a classic experiment, Ioffe of the Soviet Union showed one important way to suppress this instability. In his experiment Ioffe added around his plasma chamber six longitudinal conductors, carrying currents in opposite directions, pairwise, to the mirror coils. These "Ioffe bars" created a non-axially symmetric magnetic field which was characterized near the chamber wall by nested closed surfaces of magnetic field intensity, the field increasing from one surface to the next. In this way all MHD instabilities were suppressed, since in attempting to expand in any direction the plasma would have to move "uphill" on a magnetic pressure gradient. This principle, i.e., of the "magnetic well" would thereafter become a part of almost all mirror research, and would be adopted, where possible, in other branches of magnetic fusion research. A quadrupole version of the mirror magnetic well is shown in one of the drawings in Fig. (1).

Since the time of the Ioffe experiment additional methods have been found to suppress MHD instabilities, but the magnetic well still remains the most powerful one. These other methods include: taking advantage of so-called finite-orbit effects [8] (the difference in size of ion and electron orbits in the plasma, leading to drift motions that help to stabilize), and, stabilizing an otherwise unstable plasma in an axially symmetric mirror field by electrical conduction, along the field lines, to stabilizer cells at the ends. In these ways it has been found possible to do something not yet fully possible in toroidal systems, i.e. to eliminate the entire class of MHD instabilities, even at high plasma beta (plasma pressure approaching the confining magnetic pressure in magnitude).

In the historical development of mirror systems there was one more hurdle that was encountered, and overcome, in the march toward the fusion goal. This was the problem of the so-called "microinstabilities", high frequency instabilities of the wave-particle type that fed from the departures of mirror-confined plasma velocity-space distributions from an isotropic maxwellian distribution. As they applied to the mirror machine these instabilities were analyzed in the 1960's and methods of controlling them were suggested. When these methods were finally experimentally tested, several years later, they proved to be successful to the point that virtually quiescent plasmas, stable to both MHD and microinstabilities, were produced. These plasmas had loss times that agreed well with the "classical" values predicted by the Fokker-Planck equation, scaling with ion energy in the way predicted by 
theory. Still, the confinement values obtained were marginal if projected to fusion power conditions, leaving still open the question of how to increase the $Q$ of conventional mirror machines, where scalingwith-size does not occur as it does in toroidal systems.

At this point in mirror research (mid 1970's) one of the most spectacular successes of the mirror program occurred, in the 2XIIB experiment at the Lawrence Livermore National Laboratory. In this experiment, performed by $F$. Coensgen and co-workers $[9,10]$, a hot plasma was created in a quadrupole mirror cell by means of a large array of neutral beam sources impacting on a "seed plasma" that ionized them. The combination, a low density plasma stream and the trapped high energy deuterons, was found to be stable against both MHD and microinstability modes, and to have a beta value that approached the limiting magnetic pressure of the confining field. Furthermore, the confinement time was found to agree well with the theoretically predicted values obtained from F-P code calculations, further proof of the low levels of turbulence present in the plasma. The ion temperature, $20 \mathrm{keV}$ and plasma density, greater than $10^{14} \mathrm{~cm}^{-3}$, together represented a record achievement at the time.

The successes in 2XIIB had the effect of focussing the attention of the fusion community on the mirror approach, with the attendant programmatic pressures that that attention implied. It meant that mirror researchers had to intensify their search for ways to improve the marginal $Q$ of simple mirror systems, while at the same time paying attention to projected costs for mirror-based fusion systems. At the time there were at least three avenues to the goal visualized.

One of these ways, one that had been suggested and studied many years earlier [11,12], was to employ many mirror cells in series, arranging matters so that particles escaping from the innermost cell would be successively trapped in the outer cells, thereby achieving a confinement time that increased as the square of the number of cells, in this way regaining a scaling analogous to the quadratic scaling with size of closed systems. The difficulty, at that time, with the multiple-mirror idea was that it seemed to require such high plasma densities, and/or such long cells (to achieve collisional retrapping in each cell) as to make the entire system impractical. Later ideas [13] were aimed at alleviating this problem, but have not been experimentally tested.

Another approach, suggested in 1989 [14] and investigated at Livermore, came close to an approach we will later be discussing in this 
article. This was to devise a direct conversion system that could recover, at high efficiency, the energy of ions, both reaction products and of unburned fusion fuel, escaping through the mirrors. If coupled to a high efficiency injection system the combination might lead to a practical fusion plant. This approach was also deemed at the time to be marginal economically.

In 1976 there arose, independently, and at two different sites, a new approach to the problem - the tandem mirror idea. Dimov and coworkers at Novosibirsk [15], and Fowler and Logan [16] at Livermore came up with an improvement on an older idea of Kelly at Oak Ridge. The idea, a novel variant on the multiple mirror idea, was to have a long central cell in which fusion took place, flanked on either end with short mirror cells within which electrostatic potentials existed that electrostatically "plugged" the mirrors, reflecting back ions that would otherwise have escaped through the innermost mirrors. The overall ambipolar potential of the system would confine the electrons as usual, so that good confinement of both ions and electrons could be achieved. By making the central cell long compared to the end cells it was believed that the power required to sustain the plasmas in the end cells could be made small compared to the fusion power, so that good overall $Q$ values could be achieved. In this, first, version of the tandem mirror the increased positive potential of the plugging cells (relative to the central cell) was to be achieved by the simple expedient of maintaining a higher plasma density in those cells than that in the central cell. Mirror theory then predicted, and experiment confirmed, that the plugging potentials would appear.

The tandem mirror idea gave a new lease on life to the mirror approach. In a relatively short time tandem mirror experiments were built, at Livermore (the TMX experiment), in Novosibirsk (the AMBAL experiment), in Tsukuba, Japan (the Gamma experiment), and elsewhere. The basic principles involved were proved out, in plasmas of modest temperature and density, and a data base was begun to be assembled. However, despite the initial success of TMX in demonstrating mirror plugging, the pressure was still on for improvements that could lead to better economic projections for mirror systems. It was thought that the need for higher plasma densities in the plugging cells as compared to the central would represent too high a cost penalty in a power plant scenario. These pressures led to the invention, by Baldwin and Logan [17], of the "thermal barrier" concept, a technique employing microwave heating of electrons in the plug cells that resulted in plugging potentials 
generated in a plasma whose density was lower than that in the central cell, with important predicted economic advantages.

Although the thermal barrier idea was successfully tested, both in TMX-Upgrade and in the Gamma 10 experiment at Tsukuba, and a very large tandem mirror experiment at Livermore, MFTF, was authorized and constructed, the budget axe fell on work on "alternate concepts" in the United States, with the virtual termination of all magnetic fusion efforts except those related to the tokamak. At the present time the main centers for mirror research are at Tsukuba in Japan and at Novosibirsk in Russia, where active programs on mirror-related concepts continues. Figure 2 is a schematic drawing of the large Gamma 10 experimental facility at Tsukuba University. In recent experiments radial and axial particle loss rates consistent with confinement times of a fraction of a second have been obtained, at multi-kilovolt plasma temperatures, in good agreement with theoretical predictions. As can be seen from the figure the central magnetic field in these experiment is only 0.4 Tesla, and the central plasma column is only a few centimeters in radius. Both the field and the plasma radius are much smaller than those required in a tokamak achieving comparable confinement time constants. The improved confinement properties can be attributed to Gamma 10's axi-symmetric central-cell magnetic fields, and to the demonstrated near-complete suppression of plasma turbulence that has been achieved, accomplished through control of the radial distribution of the plasma potential.

From the more than three decades of theoretical and experimental work on open-ended systems worldwide there now exists an unusually high degree of understanding, and of correlation between experiment and theory, of plasmas confined in open-ended systems. This understanding provides a solid base for the continued investigation of such systems. The possible reward: smaller, simpler, and less expensive approaches to practical fusion power than those presently foreseen for closed confinement systems.

This understanding can also be employed to make reliable predictions about the performance of a variety of mirror-related concepts. As a result of this work we can discern the special properties of open systems vis á vis closed systems and thereby better assess their potentialities for fusion power purposes. Below we give a list of these distinguishing characteristics. 


\section{III) Distinguishing Characteristics of Open Systems}

We begin this discussion by listing those characteristics of open systems of topological origin that differentiate them operationally from closed systems. The list here is closely similar to one given in a previous paper [18]. Not every embodiment (Mirror, Tandem Mirror, FRC, RFM, etc.) possesses all of the listed characteristics, but each possesses some of them.

1) Open field topology permits the formation of deep magnetic wells in which the vacuum field possesses a non-zero minimum within the confinement zone. In such fields, as shown both by theory [19] and experiment [20], plasma beta values approaching unity can be attained and all low-frequency instabilities of MHD origin are suppressed (for non-field-reversed configurations).

2) Plasma losses can be preferentially directed to occur along the field lines, out the ends (natural "diverter" action). As noted previously, in such cases there is no requirement for the radial boundary of the plasma to be near the plasma chamber wall, thus no requirement that there should exist a radial temperature gradient at the outer boundary of the plasma. This circumstance has at least two important consequences: from the physics standpoint, as noted, the possibility of eliminating (or minimizing) radial temperature gradients in the plasma can lead to the suppression of an important class of plasma instabilities, ones that are probably playing a significant role in enhancing the rates of radial transport in closed systems, such as the tokamak. From the engineering and economic standpoint, the elimination of physical contact of the plasma with the chamber wall (or with a nearby diverter surface) greatly relieves severe heat-transfer and sputtering-life problems that are endemic to closed systems. In an open system the end-exiting fields lines can be extended and spread out so that they only encounter physical surfaces that are both far-removed from the main body of the plasma and are of large area. A concomitant, and important, result of having the plasma exit at the ends is that under the right circumstances it would permit the use of direct converters of the high-efficiency electrostatic type [21], both to recover energy from charged fusion reaction products and from unburned fuel ions.

3) Open field line topology permits the introduction, conditioning, and translation of plasma entities along the field lines, in at one end and out the other. This property, first exploited in the multi-stage magnetic compression mirror experiments at Livermore [22], has many practical 
consequences. First, it would permit the separation of the regions of plasma formation, fusion reaction, and energy extraction (by direct conversion). Second, open line topology could facilitate both the fueling and the ash removal problems (heated fuel in at one end, unburned fuel ions and fusion ash out the other end).

4) Open systems can employ linear, i.e., solenoidal, magnetic fields. Such fields are highly advantageous from an engineering standpoint when it comes to achieving high magnetic fields. It has been estimated that expected technological advances in high-field superconductors and in high-strength unidirectional fiber composites (to resist purely radial forces) should, in time, permit the design of solenoidal fields with strengths in the range of 20 to $50 \mathrm{~T}$. Particularly at the high end of this range it seems not possible to envisage toroidal-geometry field coils of usual aspect ratios with fields as high as this, owing to the intractability of the 3-D force problems involved.

5) By exploiting mirror-related properties of plasma confinement it is possible both to establish and to control electric potentials within the plasma, including the possibility of maintaining electric fields parallel to the magnetic field lines in cases where it is advantageous to do so. These potentials and electric fields have various practical applications, including enhancing confinement (as in a tandem mirror) and/or controlling the direction of exit of the plasma ions or electrons to facilitate ash removal and direct conversion.

6) By contrast with toroidal systems, where particles can only exit after diffusion across the confining field, open systems can much more readily operate near to a "collisionless" state, i.e., one where collisional randomization is not complete and where the ion (or electron) distribution functions can deviate substantially from a maxwellian. There are circumstances (for example, fusion systems involving colliding ion beams) where it may be highly advantageous to be able to employ non-maxwellian distribution functions. For example, in the case of the use of the D- ${ }^{3} \mathrm{He}$ reaction, such distribution functions facilitate the minimization of parasitic D-D reactions, thus minimize neutron fluxes and thus suppress induced radioactivity in the chamber walls. Another consequence of the ability to operate with ion distribution functions that are not collision-dominated is that such distribution functions are better suited than maxwellian distributions for high-efficiency direct conversion. (The narrower the energy distribution function of the ions is, the higher the efficiency of a given electrostatic direct converter will be.) 
7) Linear systems of open geometry may lend themselves to a "modular" design approach, something more difficult, if not impossible, to achieve with closed geometry at usual aspect ratios. For example, the central section of a TM (tandem mirror) could consist of many identical linear modules, resulting in superior accessibility and ease of construction as compared to a toroidal system.

8) Open-ended systems of the mirror or the tandem mirror type, as has been demonstrated experimentally [23,24], have the ability, through their access to the emerging field lines at the ends, to control the potential of the plasma relative to the chamber walls, including control over the radial distribution of that potential. This control can be used to enhance confinement either through limiting or through inducing plasma rotation, and for the stabilization of certain classes of plasma instabilities. This situation is to be contrasted with that prevailing in closed systems where the inherent inaccessibility of interior field lines makes it difficult if not impossible to control either the magnitude or the interior gradients of the plasma potential.

9) A qualitative, non-quantifiable, characteristic of open systems, exemplified in part by the preceding list of attributes, is their flexibility. By "flexibility" we are here implying both their adaptability to a wide variety of plasma regimes (high beta, high plasma temperature, nonmaxwellian distributions, etc.) and their suitability for innovative improvements (e.g., direct conversion). By contrast, for closed systems the requirement for closure within the confinement chamber of all field lines on which containment is to be effective introduces severe constraints on the flexibility of such systems with respect to accessible plasma regimes and with respect to innovative improvements.

\section{(IV) Dealing with the issue of end losses}

Against the above-listed attributes and advantages of open system is the main disadvantage we have already cited: the need to control end losses. This one issue has dominated the research into open-ended systems almost from its initiation. It is the issue addressed by the tandem mirror idea, but that approach is not the only one that has been considered. There are, in fact, a continuum of variants of mirror-based open systems that have fusion potential, some of which we will discuss. 
While the tandem mirror concept is aimed at achieving a high plasma $Q$ value, in concert with a similar goal in the tokamak, in this paper we are addressing the possibilities inherent in low $Q$ systems, such as the simple mirror.

A key question then for simple mirror systems becomes whether it is possible to create a viable fusion power system in which the confinement time is limited to the ion-ion relaxation time. In the language of fusion power balance, this question is whether it is feasible to operate at low $Q$ values, as contrasted with those in so-called "ignited" states postulated for the tokamak $(Q=\infty)$. Under the best conditions simple mirror systems would operate at $Q$ values that are close to unity, thus would appear to be less desirable than systems with more robust $Q$ values.

There exists a modern-day example of a highly practical power generator which operates very successfully at what fusion researchers would call a very low $Q$ value. That is, the amount of power recirculated internally is large compared to the net power output. The economic practicality of this device was not achieved by the route of increasing the equivalent $Q$ value, but by increasing the efficiency of its "injector" and "direct converter" (as they would be called by analogy with a fusion power system).

The device in question is the gas turbine. Its main components are:

1. A compressor and fuel injector (the "injector").

2. A combustion chamber (the "reaction chamber").

3. A turbine (the "direct converter"), from which power is recirculated to drive the compressor through a connecting shaft.

It is a historical fact that the first attempts to get net power from a gas turbine failed, owing to the circumstance that the efficiencies of the compressor and the turbine were too low to permit a net power output. Those first tests had to be carried out using an electric motor connected to the power shaft in order to make up for the (negative) power output of the gas turbine. Only through continued improvement in the efficiency of the compressor/ turbine system was it possible to make the gas turbine into a practical device. Even so, modern gas turbines typically operate with recirculated powers that are 3 to 4 
times greater than their net shaft output power. Yet for many applications they are eminently satisfactory and useful power generators.

We here suggest that there exists a possible development path for fusion that parallels that of the gas turbine. That is, one where the emphasis is on the twin goals of achieving an "adequate" $Q$ value (which actually in principle might be smaller than unity), while at the same time the technology of injection and direct recovery is developed (always with an eye to cost) to the point where useful net power can be obtained from the fusion system.

It is at this point that we believe that the earlier-cited "flexibility" of open-ended systems could come to the fore. Because, as also noted earlier, open systems are uniquely well adapted for ease of injection and for ease of coupling to a high-efficiency direct converter, they are prime candidates for the pursuit of the low-Q option.

It must be admitted that there is a certain element of dé-ja $v u$ in what has just been said. In a paper given in the 1976 I.A.E.A. Fusion Conference, Moir et al. [25], describe a reactor-study example of a simple mirror system using exactly the approach described above. Though one would not claim that that particular design would represent a useful power system, the general combination (some form of openended system fed by high-efficiency injectors and coupled to a highefficiency direct converter) could form an economically attractive power system, even if the fusion $Q$ value was not far different from unity.

In the sections to follow we will cite some examples of openended systems that exploit the low $\mathrm{Q}$ concept. That is, they minimize the plasma confinement time requirements through shifting the developmental burden to plasma injection and energy recovery efficiency issues.

\section{V) A Low-Q Example: A D-3 He "Linear Collider"}

As a hypothetical example of a $D-3 \mathrm{He}$ fusion power system where the intent is to suppress, to the largest degree possible, the rate of satellite D-D reactions we offer some elementary calculations to define what could be called a fusion "linear collider". The present example is based on extensions of a concept described in a previous publication [26]. 
We use the designation "collider" to denote a linear, collidingbeam, fusion power plant where the plasma and system parameters are such as to yield net power from the one-pass collision between interpenetrating beams. Stripped to essentials this system would consist of a long, graded-field, solenoid with coaxial injectors and direct converters. Time-synchronized plasma columns consisting of monoenergetic ions (helium-3 ions and deuterons) are repetitively launched so as to collide with each other near the midpoint in the solenoid. During the interpenetration of the two beams $\mathrm{D}-3 \mathrm{He}$ reactions would occur, the energy from which (together with that from unreacted beam ions) would be recovered by the direct converters. At the same time the "temperature" of the the ions of each beam would begin to grow as they scatter against the ions of the other beam and with each other. However, since the energy release per $\mathrm{D}-{ }^{3} \mathrm{He}$ fusion reaction is much higher than the total kinetic energy of the beam ions, the mutual interaction time of the two beams can be limited to a time much shorter than for complete burn-up, provided that the efficiency of the injectors and the direct converters is high enough.

By maximizing the efficiency of injection and energy recovery the burn-up fraction required for net energy production could be lowered to the point that collisional heating of the deuterons would be minimal. This result would in turn insure minimal production of $D-D$ neutrons per megawatt-year of fusion power.

A schematic power flow diagram for the linear collider is shown in Fig. (3). Taking into account the beam kinetic energies, $W_{1}$ and $W_{2}$, the fusion energy release $(18.3 \mathrm{MeV}), \mathrm{W}_{\text {fus }}$, the burn-up fraction, $\mathrm{f}$, and the injector and direct converter efficiencies, $\eta_{i}$ and $\eta_{R}$, there results an equation for the "enhanced $Q$ " of the system, $Q_{E}$, where the condition $\mathrm{Q}_{\mathrm{E}}>1$ is required for net power.

$$
Q_{E}=\left\{\frac{f W_{\text {fus }}}{W_{1}+W_{2}}\right\}\left\{\frac{\eta_{i} \eta_{R}}{1-\eta_{i} \eta_{R}}\right\}>1 \text { for net power }
$$

At this point in the discussion there exists a branching point with respect to the approach we may follow. One such approach would be to inject high density plasma streams from both ends of the system, timed to collide in the central, highest field, region of the solenoid. The second approach, which we will also briefly discuss, would be to inject, from one end, co-moving velocity-modulated ion beams whose relative 
energy in the moving frame corresponded to the maximum of the fusion cross-section. Here one counts both on the converging magnetic field and the klystron-like bunching effect to achieve a high density in the interaction region, followed by decompression and energy recovery (at low plasma density) in a direct converter.

\section{Example A: Simple Linear Collider}

We will first consider the former of the two approaches, high density colliding beams, injected from opposite ends of the solenoid.

The maximum point in the $\mathrm{D}-{ }^{3} \mathrm{He}$ reaction probability occurs at an energy of the $\mathrm{D}$ relative to the $3 \mathrm{He}$ ion of about $500 \mathrm{keV}$. From this fact it follows that for colliding beams the energy of each beam should be about $125 \mathrm{keV}$ to yield that value of relative energy. This circumstance gives a factor-of-two advantage of colliding beams over a reacting plasma in calculating the fusion power balance. Another factor of two advantage for colliding beams over a reacting plasma comes from the fact that every primary ion-ion collision carries with it the possibility of a D-3 $\mathrm{He}$ reaction, whereas in the usual plasma case (with a $50-50 \mathrm{D}-{ }^{3} \mathrm{He}$ fuel mixture) only half of the ion-ion collisions are of the "right" kind. These factors of two plus the circumstance that by design the ion-ion collisions between $\mathrm{D}$ and ${ }^{3} \mathrm{He}$ occur at the peak of the fusion crosssection help to alleviate the formidable task of achieving net power from a single-pass colliding beam situation.

The burn-up fractions that are required can be evaluated from Eq. (1) for an assumed parameter set as follows: Take $\mathrm{W}_{1}=\mathrm{W}_{2}=125 \mathrm{keV}$, $\eta_{i}=\eta_{R}=0.9$ (direct conversion efficiencies approaching this value have been demonstrated in the laboratory [27],).

When these values are inserted in Eq. (1) there results for $Q_{E}$ as a function of the burn-up fraction, $f$ :

$$
\mathrm{Q}_{\mathrm{E}}=312 \mathrm{f}
$$

so that if the burn-up fraction is taken to be 0.01 the value of $Q_{E}$ is 3.12 . As we shall see this small a value of the burn-up fraction will allow the achievement of the objective of strongly suppressing parasitic D-D reactions, provided other quantitative requirements can be met. These requirements can be estimated by considering some plasma-related issues. 
The physics points here can be illuminated by estimating two elementary quantities for the colliding beams, (1) a "mutual-scattering mean free path," and (2) a "fusion energy burn-up fraction mean free path." Comparing these two mean free paths will provide an index of the degree to which the goal of minimizing the heating of the deuterons while still providing net fusion power can be approached. Since it ignores the effect of electron drag on the deuterons, the results obtained below overestimate the ion heating resulting from ion-ion collisions between the two beams.

The effect of ion-ion collisions in causing the growth of the "temperature" of the deuteron beam ions can be estimated from collision theory as given, for example, by Spitzer [28] for a test particle (here a deuteron) moving through a field of ${ }^{3} \mathrm{He}$ ions of low temperature (in their frame of reference). We define the ratio of the perpendicular component (effectively the temperature of the test -"type $1^{\prime \prime}-$ ion) to initial relative kinetic energy as

$$
\varepsilon=\left[\frac{\left\langle\left(\Delta v_{1}\right)^{2}>_{1}\right.}{v_{12}^{2}}\right]
$$

The collisional mean free path associated with a given value of $\varepsilon$ is then given by an expression involving the Spitzer characteristic scattering time $t_{e}$ and cross-section, $\sigma_{s}$ :

$$
\lambda_{\varepsilon}=v_{1} t_{\varepsilon}=\left[\frac{\varepsilon}{n_{2} \sigma_{s}}\right], \quad \sigma_{s}=\left\{\frac{2 \pi e^{4} Z_{2}^{2} \ln \Lambda}{W_{12}^{2}}\right\}
$$

The corresponding equation for the "burn-up fraction mean free path" is:

$$
\lambda_{f}=\left[\frac{f}{n_{2} \sigma_{12}}\right]
$$


By setting the two mean free paths equal to each other we may find an expression for the fractional heating, $\varepsilon$, as a function of the burn-up fraction, given as

$$
\varepsilon=2.2 \times 10^{-12}\left[\frac{z_{2}^{2} f}{W_{12}^{2} \sigma_{12}}\right]
$$

Putting in the values for the relative energy, $W_{12}(\mathrm{keV})$, and the fusion reaction cross-section, $\sigma_{12}\left(\mathrm{~cm}^{2}\right)$, we find the value

$$
\varepsilon=35 f
$$

The implication of this value is that if $\mathrm{f}$ is small, say .01 as in the example given earlier, then $\varepsilon$ can be substantially smaller than unity, i.e., the heating of the deuterium ions by ion-ion collisions can be minimized. We should also recognize that as the two beam bunches collide the heating of the deuterium ions will start from a very low thermal temperature, rising to its maximum value only at the end of the interaction. However, throughout the encounter the colliding beams will be causing $\mathrm{D}-{ }^{3} \mathrm{He}$ fusion reactions at essentially the same rate throughout the process. This means that satellite D-D reactions will only occur at an appreciable rate during the latter part of the interaction. In code calculations of the beam-beam interactions [29] it was found that the fraction of neutron-producing reactions in the special case considered was only $2 \times 10^{-3}$ of the average rate of $\mathrm{D}^{-3} \mathrm{He}$ reactions.

\section{Electron Physics Considerations}

As with all other approaches to fusion, the role of the electrons in the plasma physics and the energetics of the system must be taken into account. In the colliding beam system we are here considering, when the two streams of ions interpenetrate, their accompanying electrons become intermixed. The relative motion of two oppositely directed ion streams through the resultant cloud of electrons (essentially at rest in the laboratory frame) gives rise to energy exchange between the ions and the electrons via electron drag force. Through this process the electrons may get heated to a substantial temperature, one which is relatively insensitive to the fractional burn-up, as is shown below. Furthermore, owing to their high collision rate, the electron distribution 
function will be Maxwellian. This circumstance will make it more difficult to recover their kinetic energy via direct conversion than it is for the more nearly monoenergetic ions. We will later discuss some possible ways to alleviate this situation. Nevertheless it is clear that electron heating is an issue that must be considered carefully in assessing the present scheme.

To assess the electron heating issue in a more quantitative way one can employ the following electron energy balance equation [30]:

$$
\frac{\mathrm{dT}_{\mathrm{e}}}{\mathrm{dt}}=\frac{176 \sqrt{2 \pi}}{9}\left[\frac{\sqrt{\mathrm{m}_{\mathrm{e}}}}{\mathrm{M}_{\mathrm{D}}}\right]\left[\frac{\mathrm{e}^{2} \ln (\Lambda)}{\mathrm{T}_{\mathrm{e}}^{3 / 2}}\right] \mathrm{n}_{\mathrm{D}} \mathrm{W}_{1},
$$

giving the rate of heating of the electrons when exposed to the deuteron beam at density $n_{D}$. The numerical factor on the right hand side of the equation takes into account electron heating by both ion species, provided the latter have the same energy and density. Assuming that they are exposed for a time $\tau=L / v_{D}$ (where $v_{D}$ is the deuteron velocity), and neglecting any decrease in ion energy caused by the energy transfer, with the interaction length, L, defined through the burn-up fraction, $f$, one obtains the following expression for the ratio of the final electron temperature to the ion energy:

$$
\frac{T_{e}}{W_{1}}=\left[\frac{440}{9 \sqrt{2 \pi}} \frac{\sigma_{s}}{\sigma_{12}} \mathrm{f}\right]^{2 / 5}
$$

The energy transferred to electrons per ion is equal to $(9 / 4) \mathrm{T}_{\mathrm{e}}$. The numerical factor, $9 / 4$, takes into account the fact that the average electron carries an energy equal to $(3 / 2) \mathrm{T}_{e}$, and that there are $3 / 2$ electrons per ion ion (owing to the presence of the ${ }^{3} \mathrm{He}$ ions). Denoting the energy conversion efficiency for electrons by $\eta_{e}$, one readily finds the following generalization of the equation for $Q_{e}$ :

$$
Q_{E}=\frac{f W_{\text {fus }}}{\left(W_{1}+W_{2}\right)} \frac{\eta_{i} \eta_{R}}{1-\eta_{i} \eta_{R}+\xi f^{2 / 5} \eta_{i}\left(\eta_{R}-\eta_{e}\right)}
$$


where

$$
\xi \approx(9 / 4)\left(20 \sigma_{s} / \sigma_{12}\right)^{2 / 5}
$$

To maintain the energy balance in the presence of electron heating it would be necessary to achieve good efficiency in the recovery of energy from the electrons. A preliminary estimate indicates that values of $\eta_{e}$ of 0.7 to 0.8 would probably be sufficient to satisfy this requirement.

\section{Plasma Column Length}

We now consider a very demanding aspect of the linear collider idea - the plasma densities and associated plasma column lengths that would be required if "single-pass" operation is demanded. It is a straightforward matter to estimate the plasma density/length requirement as a function of burn-up fraction. Figure (4) presents a plot of this relationship for two different primary beam densities (the same for each beam).

The mental picture of the linear collider that emerges from the numbers on the plots in Figure (4) is that of a very long, small bore, high field solenoid, with injectors and direct converters located at each end, the whole assembly being located in a kilometer-length tunnel below the surface of the earth. There immediately comes to mind the particle colliders and TeV-energy ion accelerators in use or under construction by particle-physics researchers. Their devices also consist of high-field superconducting magnets located in deep-lying tunnels. One such facility is the completed LEP at CERN, with a 27 kilometer long tunnel. Another could be the (now abandoned) project of the SSC accelerator in Texas, which was to have a 80-kilometer tunnel. It would not therefor seem unreasonable to visualize $\mathrm{D}-3 \mathrm{He}$ ion colliders with lengths of, say, 10 kilometers, assuming that the cost of a small-bore, superconducting, solenoidal magnet this long is economically acceptable.

Since the fusion power density for ion beams colliding with energies corresponding to the maximum point of the $\mathrm{D}-{ }^{3} \mathrm{He}$ cross-section is approximately $200 \mathrm{~kW} / \mathrm{cm}^{3}$ at a beam density of $10^{16} \mathrm{~cm}^{-3}$, the diameter of the plasma column within the collider could be quite small, of order a centimeter or two, even assuming operation at a fairly low duty cycle. However, in order to suppress "firehose" and related 
streaming instabilities in the propagating and colliding beams the magnetic field must be high. A rough estimate of this requirement is that the energy density of the confining field should exceed the kinetic energy density of the beams, implying magnetic fields in excess of $20 \mathrm{~T}$ for the cases we have considered here. As noted earlier in this report, if one is dealing with simple solenoids it is not out of the question to consider such fields for future systems, considering developments now underway.

Example B: Velocity-Modulated Co-Injected Beams

To alleviate the technical requirements on the injector system implied in Example $A$ an alternate approach has been considered $[31,32]$. Collinearly injected ion beams are to be introduced at one end of the solenoid, where the field is very low compared to its value at the midplane. These beams, derived from ion sources with accelerating grids, are to be velocity modulated so as to bunch at the point of highest field. In this way both radial and axial compression are accomplished transiently, up to densities sufficient to produce the required (small) fractional burn-ups needed for net power. Since the ion energies produced by an accelerator-grid ion source are precisely known (within a thermal spread of order $1 \mathrm{eV}$ from the source plasma) it may be possible to take advantage of this fact to achieve net power at even lower fractional burn-up values than those assumed in Example A. The reason for this circumstance is that the theoretical efficiency of the electrostatic direct converter improves directly with reduction in the spread of energy of the ions that are collected. That is to say one can express the efficiency of this type of converter by the expression:

$$
\eta_{D C}=1-\varepsilon\left\{\frac{\Delta W}{W_{1}+W_{2}}\right\} \quad \varepsilon \approx \frac{1}{N} \quad N=\text { Number of stages }
$$

However, if the initial energy spread of the beam is small, then the incremental energy spread (caused by collisions with other ions in passing through the reaction region) is given by the density-dependent expression:

$$
\Delta \mathrm{W}=\left[\mathrm{n}_{1} \mathrm{n}_{2} / \mathrm{n}_{1}+\mathrm{n}_{2}\right]\left[\sigma_{\mathrm{s}} \mathrm{v}_{12} \mathrm{t}_{0} \mathrm{~W}_{12}\right]
$$


The mutual scattering cross-section, $\sigma_{s}$, may again be estimated from Spitzer theory:

$$
\sigma_{s}=\left\{\frac{2 \pi e^{4}\left(Z_{1}\right)^{2}\left(Z_{2}\right)^{2} \ln (\lambda)}{\left[W_{12}\right]^{2}}\right\}
$$

Note now that both the energy spread and the fractional fusion burn-up depend in the same way on the plasma density, so that in the limit $\eta_{i} \rightarrow$ 1 and $\varepsilon \Delta W \ll W_{0}, Q_{E}$ becomes independent of the beam-beam interaction time, $t_{0}$ :

$$
\mathrm{Q}_{E}=\left\{\frac{\sigma_{12} \mathrm{~W}_{12} \mathrm{~W}_{\text {fus }}}{\left[2 \pi \mathrm{e}^{4}\left(\mathrm{Z}_{1}\right)^{2}\left(\mathrm{Z}_{2}\right)^{2} \ln (\lambda)\right] \varepsilon}\right\}\left\{\frac{1-\frac{\varepsilon \Delta \mathrm{W}}{\mathrm{W}_{0}}}{1+\left[\frac{1-\eta_{i}}{\eta_{i}}\right] \frac{\mathrm{W}_{0}}{\varepsilon \Delta \mathrm{W}}}\right\}
$$

Putting in numerical values for the constants gives:

$$
\mathrm{Q}_{\mathrm{E}}=\left\{3.8 \times 10^{17} \frac{\sigma_{12} \mathrm{~W}_{12} \mathrm{~W}_{\text {fus }}}{\left(\mathrm{Z}_{1}\right)^{2}\left(\mathrm{Z}_{2}\right)^{2} \varepsilon}\right\}\left\{\frac{1-\frac{\varepsilon \Delta \mathrm{W}}{\mathrm{W}_{0}}}{1+\left[\frac{1-\eta_{\mathrm{i}}}{\eta_{\mathrm{i}}}\right] \frac{\mathrm{W}_{0}}{\varepsilon \Delta \mathrm{W}}}\right\}
$$

(all energies are in $\mathrm{keV}$ )

(We have neglected here the effect of electron heating, assuming that the efficiency of the recovery of the energy of the electrons is sufficiently high.)

The maximum value of $\mathrm{Q}_{\mathrm{E}}$ occurs at the maximum value of the product of the fusion cross-section and the beam-beam relative energy: 
For D-T: $\sigma_{12} \mathrm{~W}_{12}(\max )=550 \mathrm{keV}$-barns

$$
\text { Take } \mathrm{W}_{\text {fus }}=4.5 \mathrm{MeV}+(.35 \times 14 \mathrm{MeV})=9.4 \mathrm{MeV}
$$

For $\mathrm{D}-{ }^{3} \mathrm{He}: \sigma_{12} \mathrm{~W}_{12}(\max )=420 \mathrm{keV}$-barns

Inserting these results into eq. [15] one finds for $D-T$ the value $\mathrm{Q}_{\mathrm{E}}(\max )=[2.0 / \varepsilon]$, and for $\mathrm{D}^{3}{ }^{3} \mathrm{He}$ the value $\mathrm{Q}_{\mathrm{E}}(\max )=[0.8 / \varepsilon]$. It follows that for a 10 stage converter $(\varepsilon=0.1)$ the $Q_{E}$ value for $D-T$ would be of order 20 , and for $\mathrm{D}-{ }^{3} \mathrm{He}$ it would be of order 8.0. Of course, in order to achieve these favorable values it would be necessary to find a combination of ion sources, direct converter and power circuitry, and plasma density and column length that would meet the stringent requirements stated above.

Based on some previous work [33], an analytical solution to the bunching equations has been made, in order to estimate what degree of density compression might be achieved. The calculation started with a 1-D distribution function that is a solution to the Vlasov equation for an accelerated maxwellian distribution of the ions:

$$
\begin{aligned}
\mathrm{f} & =\mathrm{f}_{0} \exp \left\{-\alpha\left[\left(\mathrm{v}_{\mathrm{x}}\right)^{2}-\left(\mathrm{v}_{0}\right)^{2}\right]\right\}, & & \mathrm{v}_{\mathrm{x}}>\mathrm{v}_{0}(\mathrm{t}) \\
& =0, & & \mathrm{v}_{\mathrm{x}}<\mathrm{v}_{0}(\mathrm{t})
\end{aligned}
$$

The acceleration potential is to be modulated so that the launching velocity is given by:

$$
v_{0}(t)=\frac{v_{I}}{1-t / t_{0}}, \quad t<t_{\text {pulse }}
$$




$$
\mathrm{t}_{0}=\frac{\mathrm{x}_{0}}{\mathrm{v}_{\mathrm{I}}} \quad \alpha=\mathrm{M}_{\mathrm{I}} / 2 \mathrm{kT}_{\mathrm{i}}
$$

To evaluate the density as a function of position and time, we can utilize the fact that the distribution function is a constant along the trajectory of the ions:

$$
f(0, t)=f\left[x,\left(t-x / v_{x}\right)\right]
$$

From this requirement the density as a function of position and time can be evaluated:

$$
\begin{aligned}
& n(t, x)=\int_{v_{\min }}^{v_{\max }} f_{0} \exp \left\{-\alpha\left[\left(v_{x}\right)^{2}-\frac{\left(v_{I}\right)^{2}}{\left[1-\frac{\left(t-x / v_{x}\right)}{t_{0}}\right]^{2}}\right]\right\} d v_{x} \\
& \text { for } t>t_{p}, v_{\max }=\frac{x}{t-t_{p}} \\
& \text { for } t<x / v_{I}, v_{\min }=x / t \\
& \text { for } x / v_{I}<t<t_{I}, v_{\min }=\frac{x_{0}-x}{t_{0}-t}
\end{aligned}
$$

Fig. (5) is a plot, calculated using eq. (19), of the axial compression factor vs $\left(x / x_{0}\right)$ for the case when $t / t_{0}=0.9$. Fig. (6) shows the same quantity when $t / t_{0}=.9999$, i.e., at a time very nearly equal to the maximum compression. The final density will, of course, be given by the product of the axial compression and the magnetic compression factor. The latter factor also has limits, ones imposed by stability conditions. An estimate of such limits can be obtained by imposing the limit that the kinetic energy density of the field should always be less than the local energy density of the magnetic field (the elementary 
criterion for the avoidance of the so-called "firehose" instability). If this is done we can use the density calculations to define a "trajectory" of intensity that the magnetic field must obey as a function of $x / x_{0}$. Fig. (7) shows such a trajectory, giving the magnetic field intensity (relative to its initial value) as a function of position.

Given the magnetic intensity and the axial compression as a function of position we can then calculate a "stable trajectory" of the plasma density as a function of position along the plasma column. The results of such a calculation are shown in Fig. (8). As can be seen, density amplifications of several orders of magnitude would seem to be feasible. The issue then becomes the technical and economic one of how to design sources, direct converters and a solenoidal field that can satisfy practical constraints. Although such a design is far beyond the intent and scope of this paper, the extrapolations involved do not seem to be unattainable.

\section{(VI) Mirror-Based Approaches with Reduced Neutron Yields}

In the realm of more traditional open-ended systems, i.e., ones based on the mirror confinement of the plasma, there may also exist other schemes that allow net energy generation from $D-{ }^{3} \mathrm{He}$ fuel at low $Q$ and at low yield of parasitic D-D neutrons. One such idea was considered in a paper by Knyazev and Ryutov [34]. In this paper it was noted that the physics of open-ended systems makes it possible to maintain the temperature of the deuterons well below the energy of the ${ }^{3} \mathrm{He}$ ions, both maintaining a high rate of $\mathrm{D}-{ }^{3} \mathrm{He}$ reactions, and at the same time exponentially suppressing the rate of the parasitic D-D reactions. By using the special technique of "drift pumping" [35] one can keep the deuteron temperature as low as $5 \mathrm{keV}$, thereby reducing the neutron yield to as low a level as $10^{-5}$ of the fusion power [36]. Since the plasma $Q$ value is low, the practicality of this virtually "nonradioactive" version of a fusion power plant also depends on the development of high efficiency direct converters and injectors.

\section{(VII) The Gas Dynamic Trap - Another Example of the Flexibility of Mirror Systems}

As another example of the flexibility of mirror-based systems, we describe here briefly a device that belongs to an opposite, that is, a strongly collisional, end of the spectrum as compared to the previous 
examples. This is a so-called "gas dynamic trap" [37], a mirror system with a very high mirror ratio $(\mathrm{R} \approx 50)$ and a length $\mathrm{L}$ satisfying the condition

$$
\mathrm{L} \geq \lambda_{\mathrm{ii}}\left[\frac{\ln (\mathrm{R})}{\mathrm{R}}\right]
$$

where $\lambda_{\mathrm{ii}}$ is the mean free path with respect to scattering by 90 degrees. The significance of this condition is that implies that the length of the mirror cell is greater than the ion scattering length over a mean angle equal to the loss-cone angle, $\theta_{L C}$, defined through the relationship, equation (1):

$$
\operatorname{Sin}\left(\theta_{L C}\right)=R^{-1 / 2}
$$

Accordingly, the ion distribution function is nearly Maxwellian at all points, with a possible exception of the region near the mirror throat. In this situation the plasma lifetime is defined by analogy with the time taken for an ordinary collisional gas to escape from a vessel with a small orifice. That is, it is given by the ratio of the total number of particles $\mathrm{nV}$ contained in a vessel of volume, $\mathrm{V}$, to the gas flux $\mathrm{nv}_{\mathrm{T}} \mathrm{S}$ escaping through an orifice of area $S$. In the present case this circumstance yields the result for the confinement time as

$$
\tau \approx \mathrm{RL} / \mathrm{v}_{\mathrm{Ti}}
$$

The origin of this expression from gas-dynamic processes explains the origin of the name.

It is to be noted that the lifetime increases linearly with the mirror ratio (and not logarithmically as it does in simple mirror systems - see equation 2). Accordingly, it is desirable to increase the mirror ratio to the maximum value that is practically attainable. In this context it is important to note that the gas-dynamic trap can be made to be MHD-stable even with axi-symmetric fields. This possibility is based on the fact that the plasma density and pressure in the mirror and immediately beyond is approximately the same as in the central cell. Thus it is that this outer region, where the field lines are concave toward the axis (field increases radially) gives an appreciable positive contribution to MHD stability. By making this line curvature large enough one can assure MHD stability of the entire plasma. This 
particular approach has been confirmed experimentally on the GDT device at Novosibirsk [38]. As noted earlier, the axial symmetry of the mirror coils allows the possibility of reaching much higher field strengths than that that is possible with non-axi-symmetric coils. As the mirror coils can be made quite short, and since the plasma diameter in the mirror throat will be relatively small $(10-15 \mathrm{~cm}$.), it is quite conceivable that magnetic fields up to 45-50 Tesla will prove to be feasible to attain in practical systems.

A fusion power system based on the GDT would operate in a steady-state regime. Plasma and heat losses through the mirrors would be compensated by the injection of neutral beams and/or by pellet injection, in combination with RF heating. On the whole, this system is attractive, both in its simplicity and in the reliability of the confinement means (tested experimentally on a moderate-scale device [38]). Unfortunately, as with the linear colliders, this simplicity has to be paid for by the fact that a GDT fusion power plant would be relatively long, of order 2 to 3 kilometers. However, we should note that practically the entire length of the confinement chamber has only a weak field (approx. 1.5 Tesla). In the final analysis then, economic (and not doctrinaire) arguments will determine the potential of the GDT for fusion power systems.

\section{(VIII) Conclusion}

To restate the premises on which this article was written, they were as follows: First, in the search for approaches to fusion that can lead in long run to economic and environmentally attractive fusion power systems the class of open-ended magnetic fusion systems has some special advantages. Second, that low-Q systems have advantages that have not been fully appreciated in the past. One such advantage is that such systems put far less demand on the confinement properties of the magnetic field, instead shifting the emphasis to the solution of some well-defined technological problems.

Another consequence of pursuing the low-Q option is to allow regimes in which the plasma behavior is more nearly governed by classical processes and less by turbulence-related ones, thereby introducing a higher degree of predictability on the basis of theory and simulation. This contention has been borne out, for example, in the pursuit of the mirror approach, where plasmas not dominated by 
turbulent transport processes have been achieved in a wide variety of experiments.

Finally, the examples of possible fusion systems given above have been given, not in the spirit of "proposals", but rather in the spirit of pointing out the wide spectrum of opportunities that exists in openended systems for novel approaches to the fusion problem, particularly as regards ones with what would be regarded as very low $Q$ values relative to the ignited tokamak, for example. The effect of taking the low $Q$ route, as noted, both shifts the emphasis from containment to technology issues, and at the same time opens up regimes, for example, ones with very low parasitic neutron-producing reactions, that are completely inaccessible to approaches that require a high $Q$ value.

*Work performed under the auspices of the United States Department of Energy by the Lawrence Livermore National Laboratory, under Contract No. W-7405-ENG-48.

\section{References:}

(1) P. W. Terry, et. al., in: Plasma Physics and Controlled Fusion 1992, (I.A.E.A. 1993) Vol 2., p. 313

(2) C. Störmer, Z. f. Astrophys., 3, 31, 227 (1931)

(3) J. A. Van Allen, C. E. Mc Ilwain, G. H. Ludwig, J. Geophys. Res. 64, 271 (1959)

(4) T. G. Northrop, E. Teller, Phys. Rev. 117, 215 (1960)

(5) G. Gibson, W. C. Jordan, E. J. Lauer, Phys. Fluids 6, 116 (1963)

(6) L. G. Kuo-Petravic, M. Petravic, C. J. H. Watson, in Nuclear Fusion Reactors (Proc. Int. Conf. Culham,1969), British Nuclear Energy Society, London, 144 (1969)

(7) R. F. Post, R. E. Ellis, F. C. Ford, M. N. Rosenbluth, Phys. Rev. Lett. 4, 166 (1960)

(8) M. N. Rosenbluth, N. A. Krall, N. Rostoker, Nucl Fus. Suppl., Part I, 143 (1962) 
(9) A. W. Molvik, F. H. Coensgen, W. F. Cummins, et. al., Phys. Rev. Lett. 32, 1107 (1974)

(10) B. G. Logan, J. F. Clauser, F. H. Coensgen, et. al., Phys. Rev. Lett. 36, 1468 (1976)

(11) R. F. Post, Phys. Rev. Lett. 18, 232 (1967)

(12) G. I. Budker, V. V. Mirnov, D. D. Ryutov, JETP Lett. 14, 214 (1971)

(13) R. F. Post, X. Z. Li, Nucl Fusion 21, 135 (1981)

(14) R. F. Post, in Nuclear Fusion Reactors (Proceedings British Nucl. Energy Soc. Conf. London, 1969), Culham Laboratory, Abingdon, Oxfordshire (1969) p.88

(15) G. I. Dimov, V. V. Zakaidakov, M. E. Kishinevskiia, Sov. J. Plasma Phys. 2, 326 (1976)

(16) T. K. Fowler, B. G. Logan, Comments Plasma Phys. Controlled Fusion 2, 167 (1977)

(17) D. E. Baldwin, B. G. Logan, Phys. Rev. Lett. 43, 1318 (1979)

(18) R. F. Post in Proceedings of Course and Workshop: "Physics of Mirrors, Reversed Field Pinches and Compact Tori", Varenna, Italy, Vol. I, 51 (1987)

(19) R. J. Hastie, J. B. Taylor, Phys. Fluids 8, 323 (1965)

(20) D. L. Correll, J. F. Clauser, F. H. Coensgen, et. al., Nucl. Fusion 20, 655 (1980)

(21) R.W. Moir, W. L. Barr, G. A. Carlson, in Plasma Physics and Controlled Fusion 1974 (I.A.E.A. Vienna 1975) Vol. 3, p. 583

(22) F. H. Coensgen, W. F. Cummins, W. E. Nexsen, Jr., A.E. Sherman, Nucl. Fusion Suppl., Part I, 125 (1962)

(23) G.F. Abdrashitov, A.A.Bekhtenev, V.V. Kubarev, V.E. Pal'chikov, V.I.Volosov, Yu.N. Yudin. In: "Mirror Based and Field Reversed Approaches to Magnetic Fusion", Proc. of the 1983 Varenna School of Plasma Physics, Monotypia Franchi, v.1, p.335, 1984. 
(24) A. Mase et al., Nuclear Fusion, 31, 1725 (1991)

(25) R. W. Moir, W. L Barr, D. J. Bender, et al., in Plasma Physics and Controlled Fusion 1976 , Vol. III, 237, IAEA, Vienna (1976)

(26) R. F. Post, J. F. Santarius, Fusion Technology, 22, 13 (1992)

(27) See Ref. 21

(28) L. Spitzer, Jr., Physics of Fully Ionized Gases, Interscience (1962)

(29) See Ref. 26

(30) NRL Plasma Formulary, NRL/PU/6790-94-265, Naval Research Laboratory, Washington DC, 1994.

(31) R. F. Post, "Exploring the Limits of the 'Low Q' Fusion Power Regime", Paper 1D18, in : Proceedings of the Sherwood International Fusion Theory Conference," 14-16 March 1994.

(32) R. F. Post, "Some Theoretical Aspects of 'Linear Collider' OpenEnded Fusion Power System", in: Proceedings of the Sherwood International Fusion Theory Conference," 3-5 April 1995

(33) K. D. Marx, C. J. Eggens, R. F. Post, JAP 48, 4215 (1977)

(34) B. A. Knyazev, D. D. Ryutov, in: "Tritium and Advanced Fuels in Fusion Reactors," Proc. of the 1988 International School of Plasma Physics "Piero Caldirola," p. 693, Editrice Copositori, 1989

(35) D. D. Ryutov, Proc. of the 2nd Wisconsin Symposium on Helium-3 and Fusion Power, (J. F. Santarius, compiler), WSCAR-TR-AR3-9307-3, University of Wisconsin, Madison, p. 121, (1993)

(36) D. E. Baldwin, in: "Mirror-Based and Field-Reversed Approaches to Magnetic Fusion," Proc. of the 1983 Varenna School of Plasma Physics, Monotypia Franchi, Vol. 1, 109 (1984)

(37) V. V. Mirnov, D. D. Ryutov, Sov. Technical Physics Lett., 5, 279 (1978)

(38) A. S. Ivanov, et al., Physics of Plasmas, 1, 1529 (1994) 


\section{Figure Captions}

Fig. (1) Schematic drawings showing the historical evolution of the mirror concept. In the upper left corner is shown the simple axisymmetric mirror. In the upper middle a quadrupole magnetic well, as produced by a "baseball" coil is shown. At the upper left is the simple tandem mirror, and at the bottom a tandem mirror with thermal barriers is shown.

Fig. (2) A schematic drawing of the Gamma 10 tandem mirror experiment at Tsukuba, Japan. Note that in its present configuration all heating and potential generation is accomplished with RF and/or microwave power. Note also that the central cell and its mirrors are axi-symmetric, leading to improved confinement as compared to nonaxi-symmetric cells as were used in some earlier tandem mirror experiments.

Fig. (3) Block diagram of a counter-streaming "linear-collider" fusion power system. An important feature is the recirculation of unreacted ions and the direct recovery of their energy at high efficiency.

Fig(4) The burn-up fraction for colliding beams, plotted as a function of the beam-beam interaction length, with plasma density as a parameter.

Fig. (5) An in-flight "snapshot", at a normalized time of 0.9 , of the beam plasma density (relative to its value at launching), as calculated by equation (19). Initial deuteron temperature: $1 \mathrm{eV}$; initial energy: 100 $\mathrm{keV}$; pulse fractional time: 0.5 .

Fig. (6) In-flight snapshot at normalized time 0.9999 , all other parameter the same as in Fig. (5).

Fig. (7) Relative variation of solenoid magnetic field along beam trajectory, as required to satisfy simple criterion for avoidance of the firehose instability.

Fig. (8) Firehose-stable density "trajectory" resulting from satisfaction of requirement on magnetic field shown in Fig. (7). 


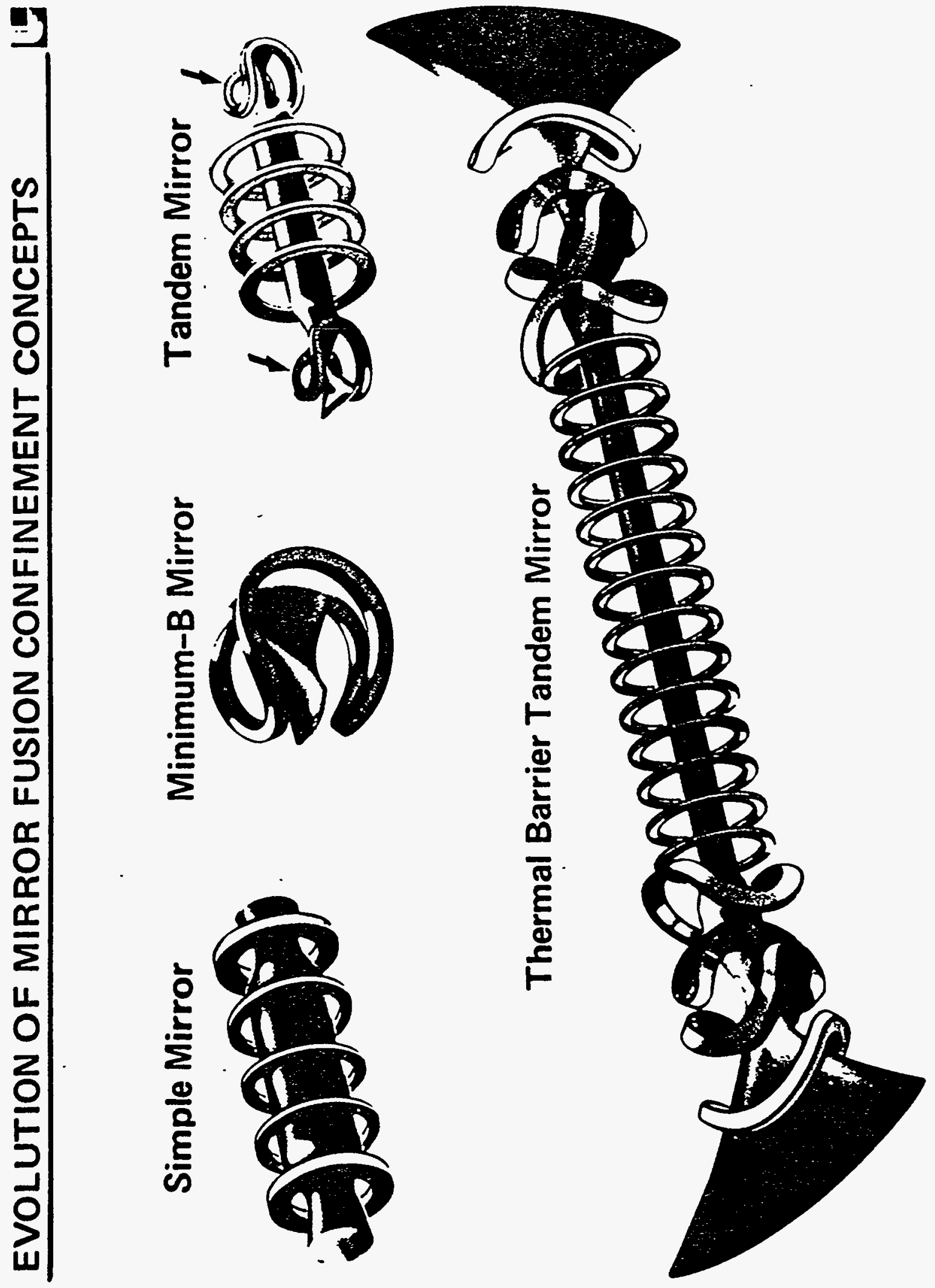




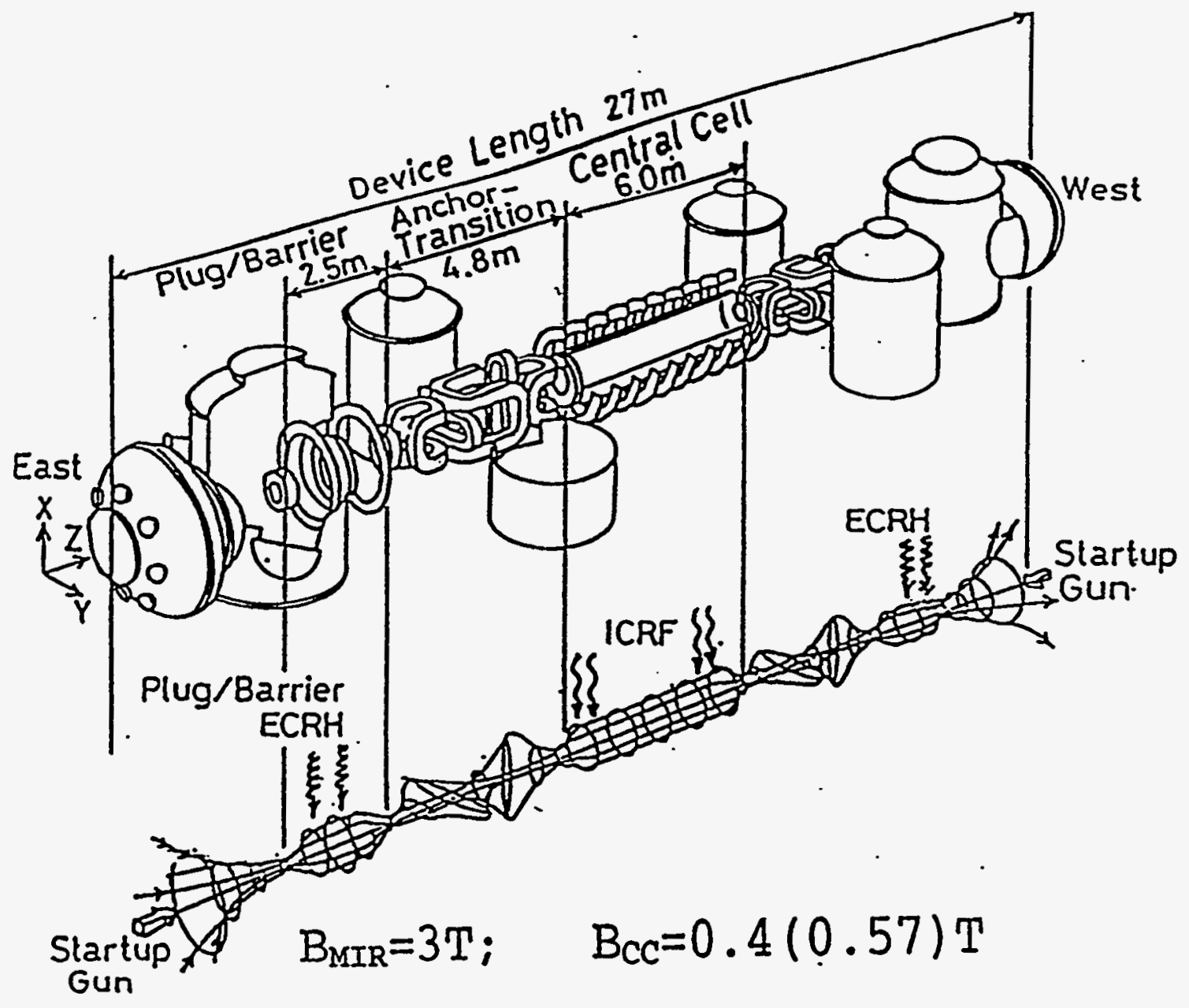




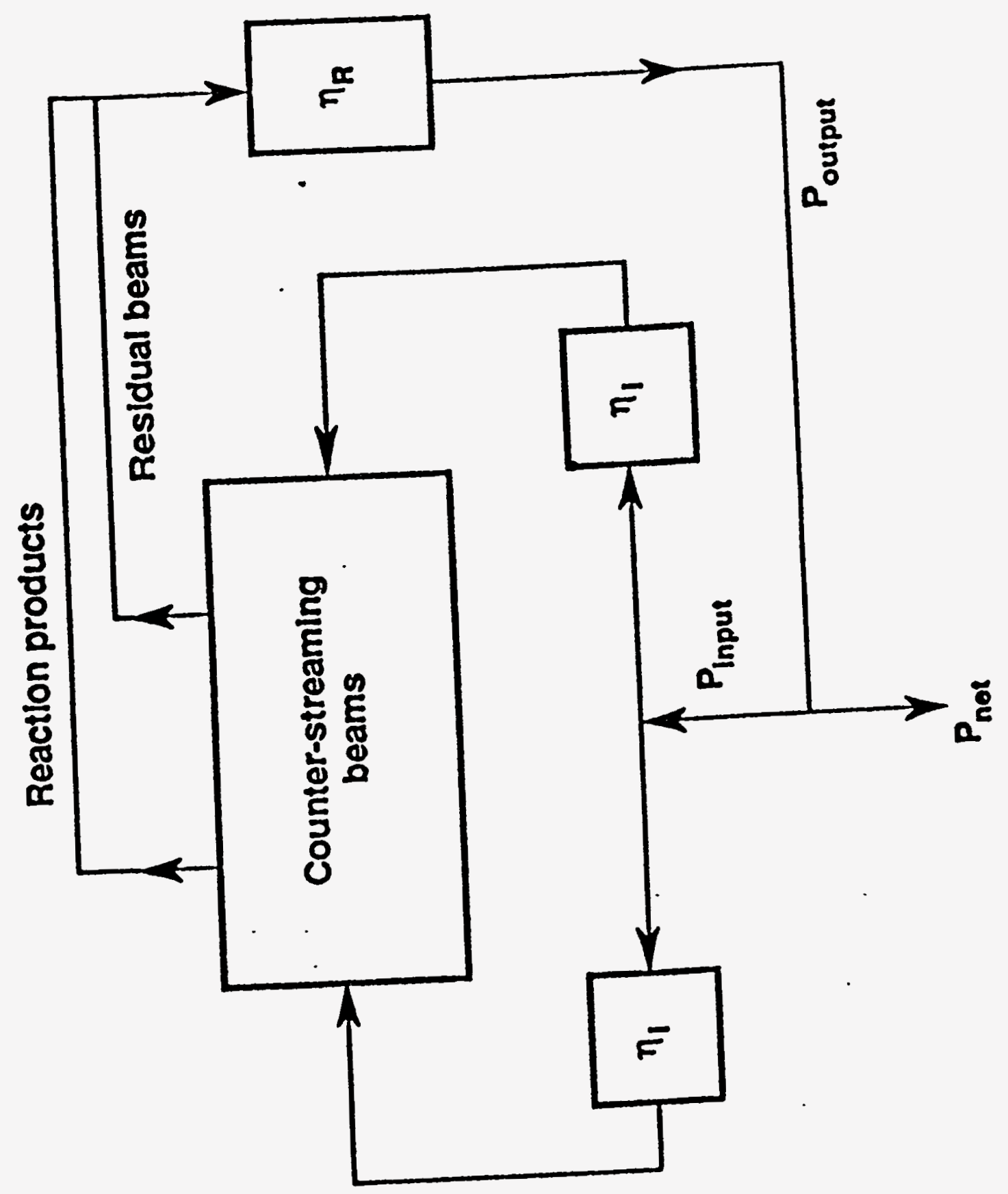




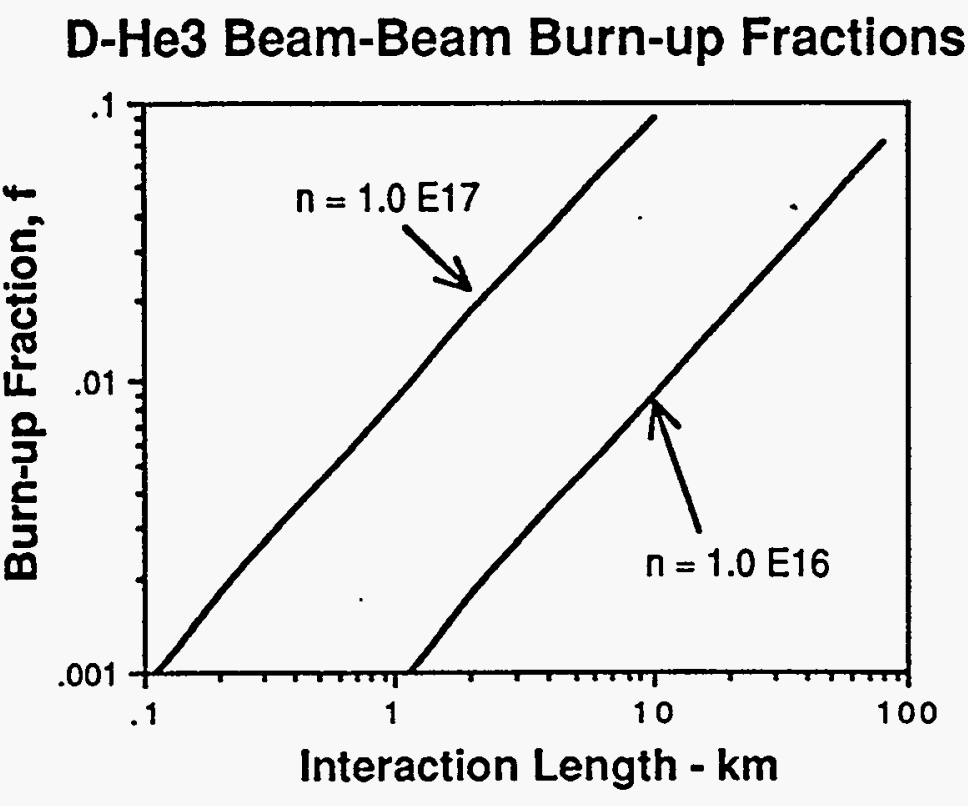


Relative Density vs $\mathrm{x} / \mathrm{xO}$

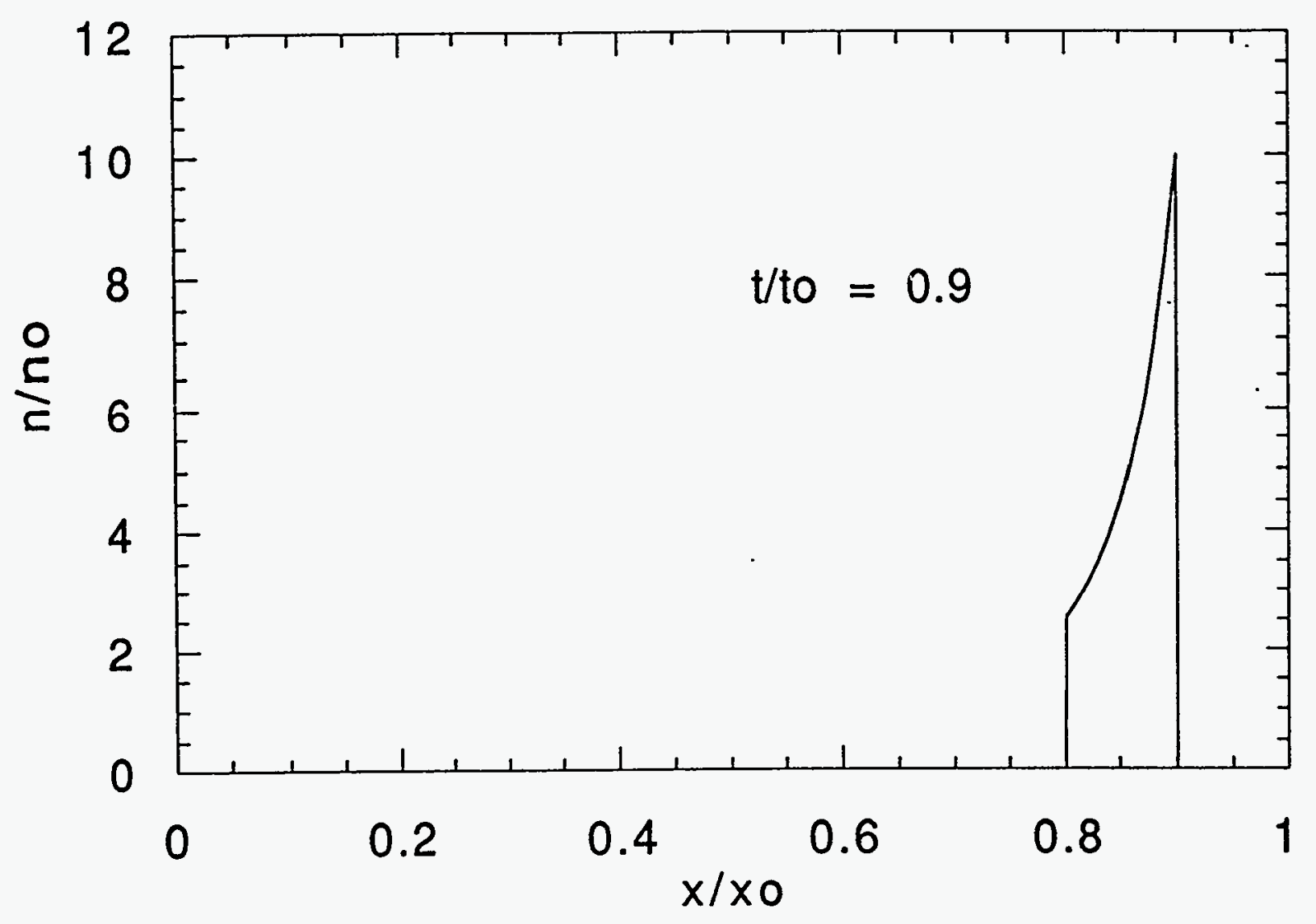


Relative Density vs $\mathrm{x} / \mathrm{xO}$

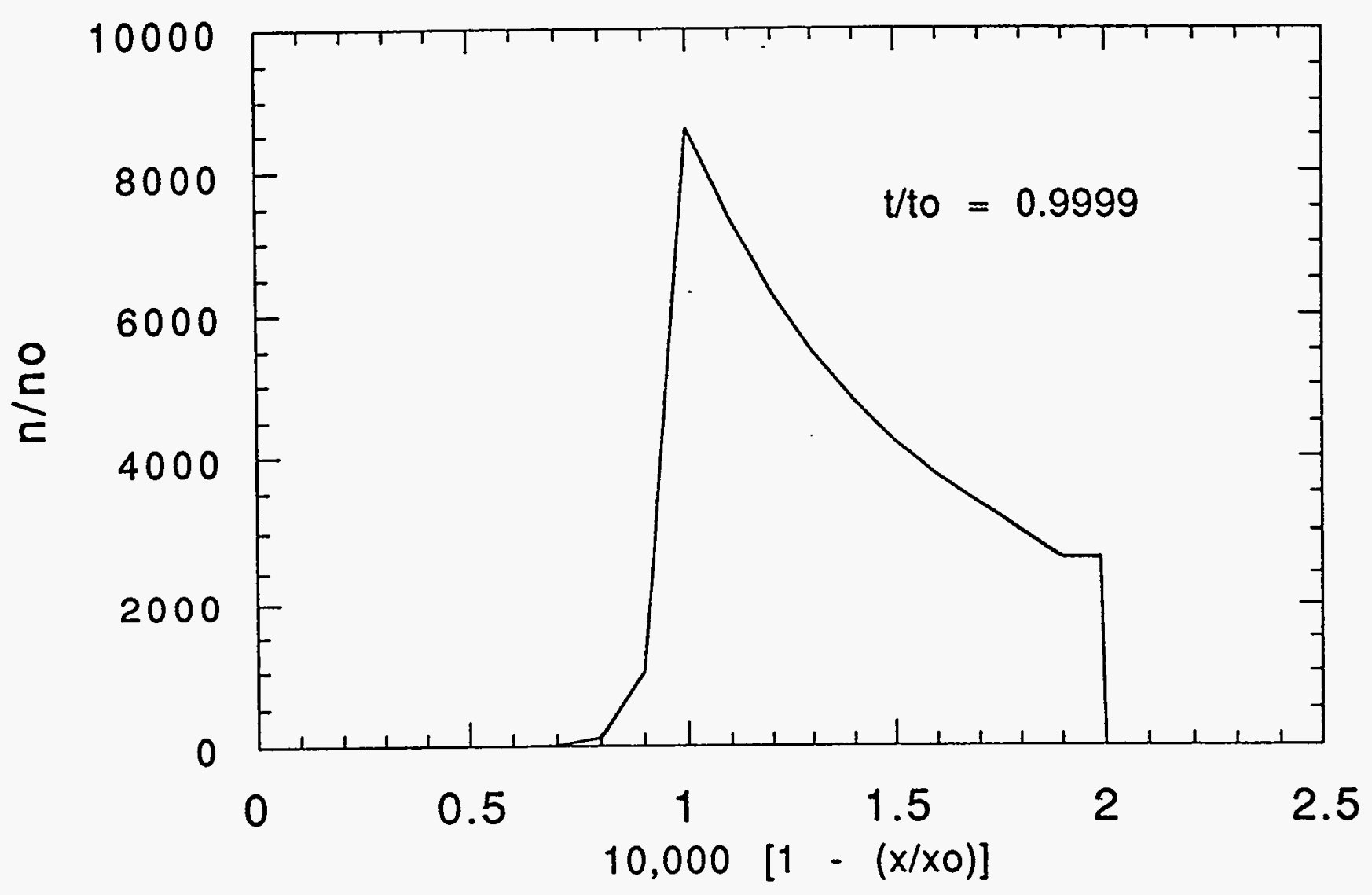


B/Bo Required for Firehose Stability vs $x / x 0$

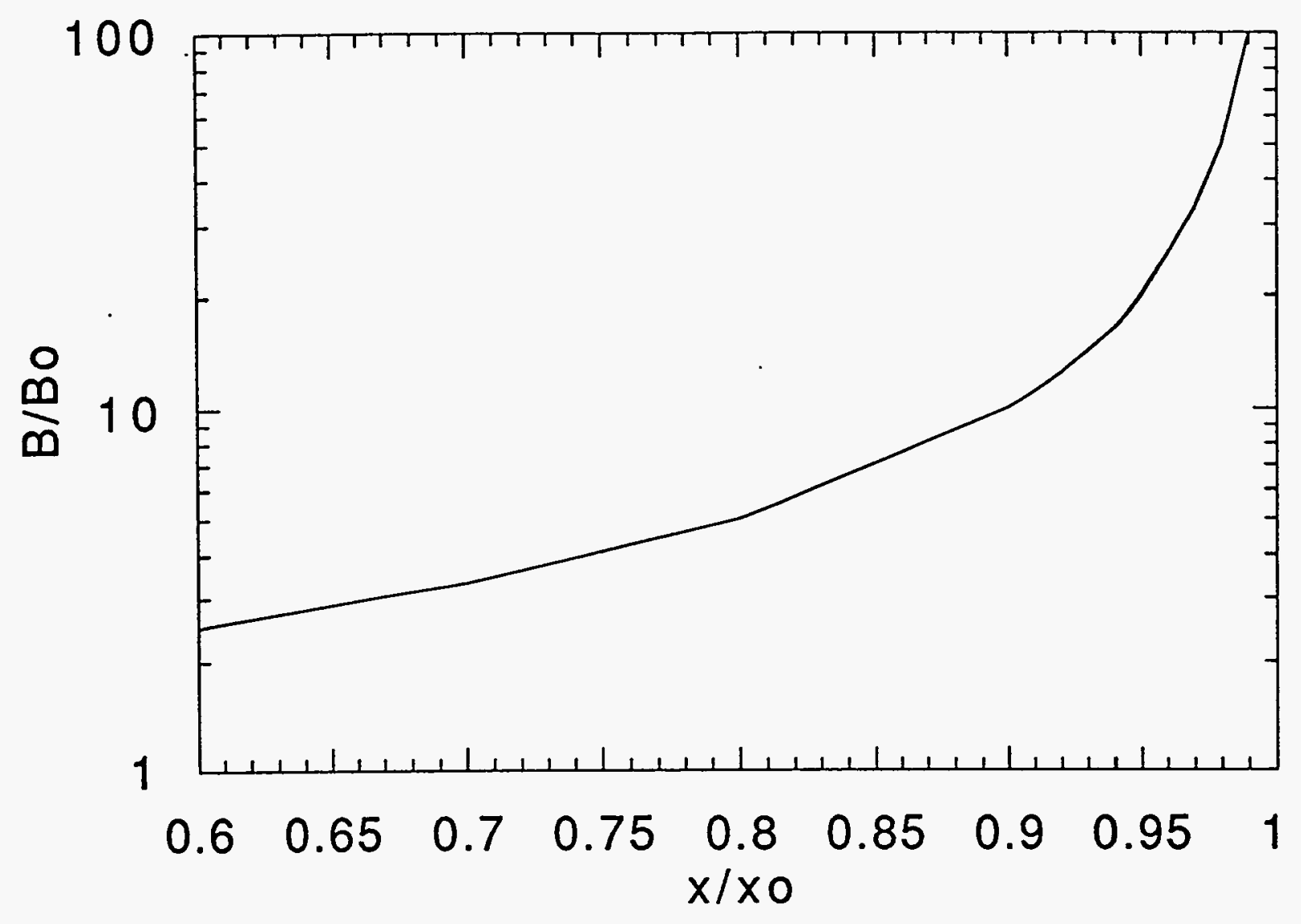


Firehose-Stable Density Trajectory vs $\mathrm{x} / \mathrm{xo}$

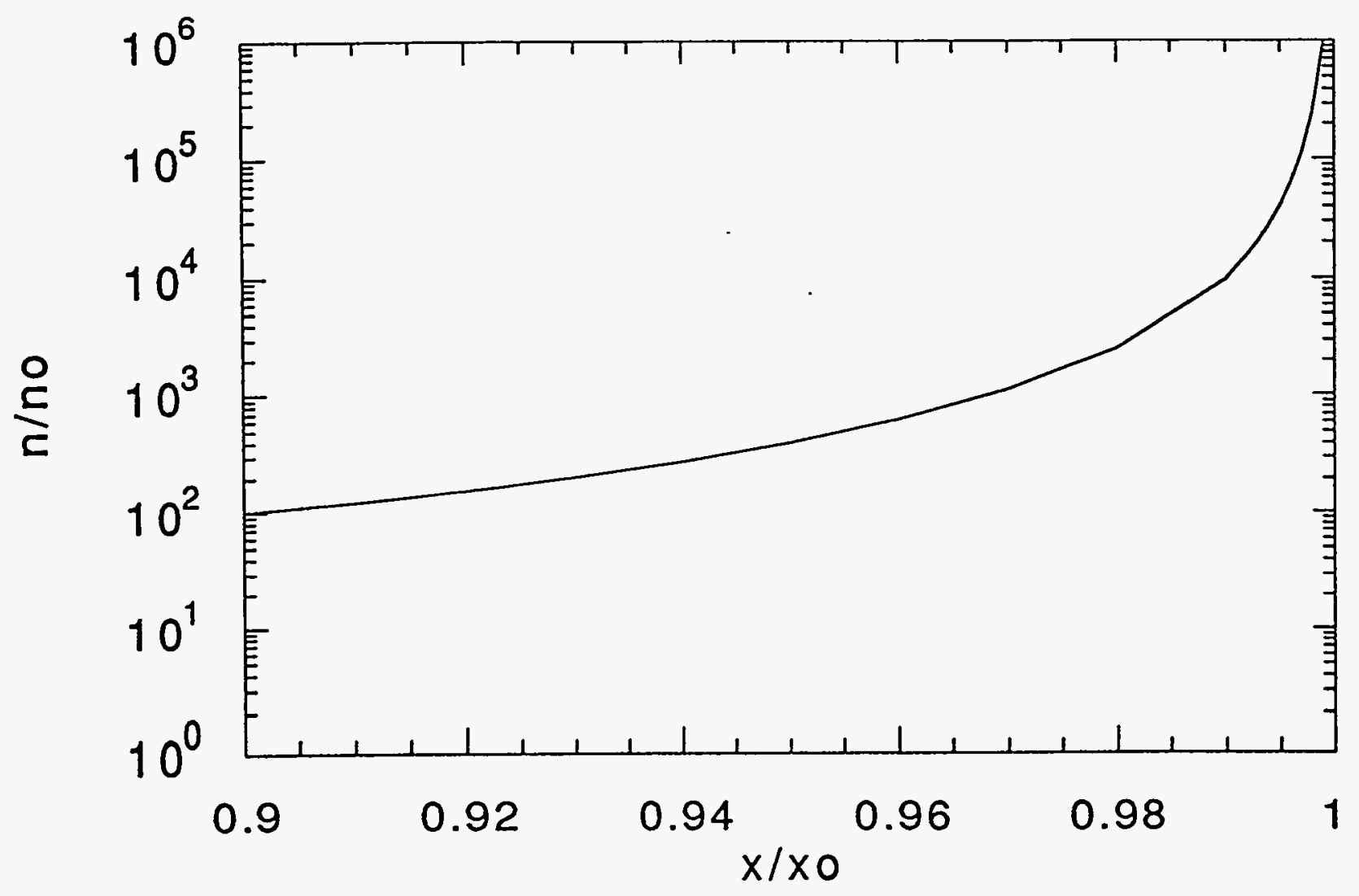

